\title{
$L 1_{0}-\mathrm{FePt}(001) / A 1-\mathrm{FePt}$ 双层交换弹簧的磁性
}

\author{
魏娟 ${ }^{1}$, 杜斌 ${ }^{1}$, 李佳芹 ${ }^{1}$, 姚雅芹 ${ }^{1}$, 陈阳 ${ }^{1}$, 李鱼辉 ${ }^{1}$, 曾道富 ${ }^{1}$, 李国庆 ${ }^{1 *}$, 张昆华 ${ }^{2}$, 闻明 ${ }^{2}$ \\ 1. 西南大学物理科学与技术学院, 重庆 400715 ; \\ 2. 昆明贵金属研究所, 昆明 650106 \\ *联系人, E-mail: gqli@swu.edu.cn
}

2016-05-11 收稿, 2016-08-10 修回, 2016-08-11 接受, 2016-12-14 网络版发表

国家自然科学基金(51262015)和国家大学生创新创业训练计划(201510635044)资助

\begin{abstract}
摘要 在加热到 $400{ }^{\circ} \mathrm{C}$ 的 $\mathrm{MgO}(001)$ 单晶基片上, 用磁控溅射法沉积 $30 \mathrm{~nm}$ 厚 $A 1$ 相 $\mathrm{FePt}$ 软磁薄膜, 在 $T_{\mathrm{a}}=\left[400^{\circ} \mathrm{C}\right.$, $\left.700^{\circ} \mathrm{C}\right]$ 温度范围进行 $6 \mathrm{~h}$ 热处理, 使其发生不同程度的 $A 1 \rightarrow L 1_{0}$ 转变. 以此作为硬磁层, 在 $100^{\circ} \mathrm{C}$ 继续生长不同厚度 的 $A 1$ 相 $\mathrm{FePt}$, 得到具有 $(001)$ 织构的 $L 1_{0} / A 1-\mathrm{FePt}$ 双层磁性交换弹簧. $T_{\mathrm{a}} \leqslant 600^{\circ} \mathrm{C}$, 硬磁层的覆盖率保持 $100 \%$, 但晶粒 尺寸有差别. $T_{\mathrm{a}}=500^{\circ} \mathrm{C}$ 和 $600^{\circ} \mathrm{C}$, 硬磁层的有序度分别为 0.61 和 0.84 , 层中 $L 1_{0}$ 相与 $A 1$ 相共存, 两相间的交换作用使 薄膜出现垂直磁晶各向异性, 磁化曲线呈方形. 矫顽力在 $T_{\mathrm{a}}=500^{\circ} \mathrm{C}$ 时接近 $5 \mathrm{kOe}$. 用 $T_{\mathrm{a}}=500^{\circ} \mathrm{C}$ 和 $600^{\circ} \mathrm{C}$ 的硬磁层做 成 $L 1_{0}(001) / A 1-\mathrm{FePt}$ 双层薄膜, 在软磁层厚度为 $20 \mathrm{~nm}$ 时, 磁化曲线仍然保持方形, 而软磁层厚度增加到 $30 \mathrm{~nm}$, 反 磁化曲线分成软磁层反转和硬磁层反转两个阶段, 表明层间交换作用长度都在 $20 \mathrm{~nm}<l_{\mathrm{ex}}<30 \mathrm{~nm}$ 范围, 是理想 $L 1_{0} / A 1-\mathrm{FePt}$ 交换弹性体系的 2 倍以上. 经过分析认为, 出现这种现象的原因在于硬磁层中 $L 1_{0}$ 相与 $A 1$ 相共存, 降低 了有效单轴磁晶各向异性能密度. 这为根据需要设计磁性弹簧的性质提供了方便.
\end{abstract}

关键词 $L 1_{0}-\mathrm{FePt} / A 1-\mathrm{FePt}$ 双层薄膜, 磁性弹簧, 矫顽力, 交换作用长度

$\mathrm{Fe}, \mathrm{Co}, \mathrm{Ni}$ 等强磁性元素与 Pt, Pd等贵金属元素结 合, 在等原子比附近形成 $L 1_{0}$ 相的硬磁合金, 具有按 原子种类逐层有序排列的面心四方 (face centered tetragonal, FCT) 晶体结构, 会产生巨大的单轴磁晶各 向异性能 $\left(K_{\mathrm{u}}\right)$, 又具有比稀土永磁材料耐腐蚀的优 点, 在计算机硬盘垂直磁记录介质、集成电路微小膜 式永磁体、磁力显微镜针尖磁性涂层等领域有广泛的 应用前景 ${ }^{[1 ~ 6]}$. 其中, $L 1_{0}-\mathrm{FePt}$ 的 $K_{\mathrm{u}}=\sim 7 \times 10^{7} \mathrm{erg} / \mathrm{cm}^{3}(1$ $\left.\mathrm{erg} / \mathrm{cm}^{3}=0.1 \mathrm{~J} / \mathrm{cm}^{3}\right)$, 室温超顺磁极限尺寸不足 $3 \mathrm{~nm}$ (按 $K_{\mathrm{u}} V / k_{\mathrm{B}} T \geqslant 60$ 计算, 满足 10 年内热扰动引起自发磁 化发生反转的概率不大于 $1 / 10^{6}, V$ 是球状磁性晶体的 体积, $k_{\mathrm{B}} T$ 是热扰动能), 器件尺寸可以更小. 饱和磁 化强度 $M_{\mathrm{s}}=\sim 1200 \mathrm{emu} / \mathrm{cm}^{3}\left(1 \mathrm{emu} / \mathrm{cm}^{3}=10^{3} \mathrm{~A} / \mathrm{m}\right)$ 较大, 做成小器件也能保证足够的信号强度 ${ }^{[7 ~ 12]}$. 但是颗粒
化薄膜的室温矫顽力 $\left(H_{\mathrm{c}}\right)$ 高达 $70 \mathrm{kOe}\left(1 \mathrm{Oe}=10^{3} / 4 \pi\right.$ $\mathrm{A} / \mathrm{m})$, 理论极限开关场为 $H_{\mathrm{k}}=2 K_{\mathrm{u}} / \mu_{0} M_{\mathrm{s}}=\sim 116 \mathrm{kOe}^{[13]}$. 矫顽力太大给应用中改变器件的磁化状态带来困难, 必须想办法降低.

1991年, Kneller和Hawig ${ }^{[14]}$ 提出交换弹簧(exchange spring)概念, 想法是将硬磁物质与软磁物质结合在 一起，使两相在界面处发生耦合，产生交换弹性作 用, 可以兼得硬磁材料的高 $H_{\mathrm{c}}$ 和软磁材料的高 $M_{\mathrm{s}}$, 提高磁能积. 软磁层厚度超过交换作用长度, 磁化反 转会由单一开关场转变为软磁相内形核成畴和硬磁 相开关两次反转 ${ }^{[15,16]}$. 用 $L 1_{0}-\mathrm{FePt}$ 做过的尝试有 $L 1_{0}$ $\mathrm{FePt} / \mathrm{Fe}$ 双层结构 ${ }^{[17 \sim 19]}$. Fe 的 $M_{\mathrm{s}}\left(\sim 1700 \mathrm{emu} / \mathrm{cm}^{3}\right)$ 比 $\mathrm{FePt}$ 的高. 但制约 $L 1_{0}-\mathrm{FePt}$ 应用的主要问题不是磁能 积小, 而是 $H_{\mathrm{c}}$ 实在太大. 只降低 $H_{\mathrm{c}}$, 希望能够避开抗 
氧化能力差的 $\mathrm{Fe}$. 使用坡莫合金 $(\mathrm{Fe}$ 和 $\mathrm{Ni}$ 组成的一种 耐蚀软磁材料), 又产生两层间晶格不匹配问题. 晶 格类型不同, 或者晶格常数相差很大, 上层薄膜生长 时外延性都不理想, 影响交换弹性作用的效果 ${ }^{[20]}$. $\mathrm{FePt}$ 合金在常温环境中还存在一种具有面心立方 (face centered cubic, FCC)晶体结构的亚稳相(用 $A 1$ 表 示, 可以认为是高温相的过冷态), $\mathrm{Fe}$ 和 $\mathrm{Pt}$ 原子随机出 现在格点位置, 不具备成分有序的特征. 在常温获得 的 FePt薄膜通常都处于 $A 1$ 相, 需要经过适当的热处 理, 才能转变为更稳定的 $L 1_{0}$ 相 ${ }^{[21,22]} . A 1-\mathrm{FePt}$ 也具有 铁磁性, 但立方磁晶各向异性能很低. $L 1_{0}-\mathrm{FePt}$ 和 $A 1-\mathrm{FePt}$ 共格生长形成同材异质界面, 就可以在纳米 维度得到一种特殊的硬磁/软磁复合材料 ${ }^{[23]}$. 用 $L 1_{0} /$ $A 1-\mathrm{FePt}$ 作为磁性弹簧, 解决了界面外延生长问题. 但评价这种复合材料的实用性, 尚需对体系的形成 条件进行更细致的调控, 分析矫顽力变化, 掌握磁化 反转机制. 特别是这种同材异质交换弹簧的层间交 换作用长度究竟与哪些因素有关, 需要从实验数据 积累和理论分析两方面进行深人探索. 可行的办法是 改变硬磁层的有序化转变程度以及两层各自的厚度.

由于(111)晶面是面心立方晶格的密堆积面(每个 原子在面内有 6 个最近邻原子), 如果在非晶基片(如 玻璃)或晶格不匹配的单晶基片(如 $\mathrm{Si}$ 或石英单晶)上 沉积 $\mathrm{FePt}$, 原子在溅射动能的推动下容易逐层密堆 积, 出现(111)织构 ${ }^{[24]}$. 而用 $\mathrm{MgO}(001)$ 单晶作为基底, $\mathrm{FePt}$ 薄膜就可以取向生长, 形成(001)织构, 从而获得 垂直磁晶各向异性 ${ }^{[25,26]}$.

本文在加热的 $\mathrm{MgO}(001)$ 基片上用磁控溅射法生 长 FePt, 然后在不同温度进行热处理, 以获得不同 $A 1$ $\rightarrow L 1_{0}$ 转变程度的硬磁层 (为了方便, 文中把硬磁层都 表述为 $L 1_{0}-\mathrm{FePt}$ ). 然后再生长完全处于 $A 1$ 相的第二 层 $\mathrm{FePt}$, 分析硬磁层有序化程度及软磁层厚度对磁 化曲线的影响, 找出影响交换作用长度的主导因素, 为 $L 1_{0} / A 1-\mathrm{FePt}$ 这种特殊磁性弹簧的应用提供实验和 理论依据.

\section{1 实验}

$\mathrm{MgO}$ 的晶格常数 $\left(a_{\mathrm{MgO}}=0.4203 \mathrm{~nm}\right)$ 比 $A 1-\mathrm{FePt}$ 的 $\left(a_{A 1-\mathrm{FePt}}=0.3841 \mathrm{~nm}\right)$ 稍大, 并都具有 $\mathrm{FCC}$ 结构. 所以 $\mathrm{FePt}$ 可以在 $\mathrm{MgO}(001)$ 基片上取向生长, 形成(001)织 构, 但膜面 ( $a-b$ 面)内会受到基片施加的拉伸应力, 导 致与 $a-b$ 面垂直的 $c$ 轴有缩短的趋势. 进行热处理后,
$A 1$ 相转变为稳定的 $L 1_{0}$ 相(变成FCT结构, $a=b=0.3905$ $\mathrm{nm}, c=0.3735 \mathrm{~nm}$ ), 在应力的诱导作用下, 缩短的 $c$ 轴 会沿着与膜面垂直的方向. 因此, 不管薄膜是否连 续, 所有晶粒或大片的晶体都具有垂直磁晶各向异 性. 第二层FePt同样沿[001]方向取向生长, 从而得到 $L 1_{0}(001) / A 1-\mathrm{FePt}$ 同材异质结.

成膜采用磁控溅射镀膜法, 背景真空度优于 $2 \times 10^{-5} \mathrm{~Pa}$, 工作时通人压强为 $2.8 \mathrm{~Pa}$ 的 $\mathrm{Ar}$ 气. $\mathrm{Fe}$ 靶和 $\mathrm{Pt}$ 靶的轴线成一定角度, 并且可以同时工作. 两靶纯 度均不低于 $99.9 \%$. 成分比例依靠溅射功率调解, 厚 度用溅射时间控制. 第一层 $\mathrm{FePt}$ 的成膜温度为 $400^{\circ} \mathrm{C}$. 成膜时加热基片, 能够提高外延生长的质量. 但如果 温度再高, 会增大薄膜的表面高低差, 使表面不够平 整, 影响膜厚的均匀性, 降低覆盖率. 生长 $30 \mathrm{~nm}$ 厚 的FePt薄膜后, 取出样品, 放人真空热处理炉进行 6 $\mathrm{h}$ 的热处理, 温度 $\left(T_{\mathrm{a}}\right)$ 在 $400 \sim 700^{\circ} \mathrm{C}$ 范围, 使薄膜发生 不同程度的 $A 1 \rightarrow L 1_{0}$ 转变. 选定厚度为 $30 \mathrm{~nm}$, 是为了 保证薄膜的覆盖率为 $100 \%$. 之后再生长一层 $A 1-\mathrm{FePt}$, 得到 $L 1_{0}-\mathrm{FePt}(30 \mathrm{~nm}) / A 1-\mathrm{FePt}(x \mathrm{~nm})$ 双层复 合薄膜. 为了能够观察到磁化曲线的明显变化, 厚度 选定 $x=20$ 和 $30 \mathrm{~nm}$ 两种典型情况. 为了防止层间扩散, 第二层的生长温度为 $100^{\circ} \mathrm{C}$. 实验前反复调整溅射时 间并用表面轮廓仪检测薄膜厚度(用油性记号笔在基 板上画线, 成膜后在酒精中进行超声波清洗, 去掉线 上覆盖的薄膜和油性物质, 形成边缘陡峻的台阶), 以提高厚度的可靠性. 用原子力显微镜 (AFM) 观察 表面形貌，用X射线衍射仪(XRD)分析晶体结构(使 用 $\mathrm{Cu}-K_{\alpha}$ 射线源, 波长为 $0.15406 \mathrm{~nm}$ ), 用振动样品磁 强计(VSM) 测量 $M-H$ 曲线 (计算磁化强度 $M$ 时计人双 层薄膜的总体积, 外磁场强度 $H$ 最大为 $\pm 20 \mathrm{kOe}$ ).

\section{2 结果与讨论}

\section{1 热处理前后FePt单层薄膜的性质}

图 1是 $\mathrm{FePt}\left(30 \mathrm{~nm}, T_{\mathrm{a}}\right.$ ) 薄膜的XRD图谱, 使用 $\theta-2 \theta$ 联动模式, 只有平行于膜面的晶面族才形成衍 射峰. 图中标出指数的峰来自 $\mathrm{FePt}$, 其他的都来自 $\mathrm{MgO}$ 基片. $\mathrm{FCC}$ 晶格的消光规律是不出现指数 $(h k l)$ 为 奇数和偶数混合的衍射峰. $T_{\mathrm{a}}=400^{\circ} \mathrm{C}$ (热处理温度与 成膜温度相同), 只观察到(200)基础峰, 没有明显的 (001)和(003)衍射峰, 说明薄膜有取向生长但处于无 序的 $A 1$ 相. 相对于标准位置, (200)峰出现的角度小幅 


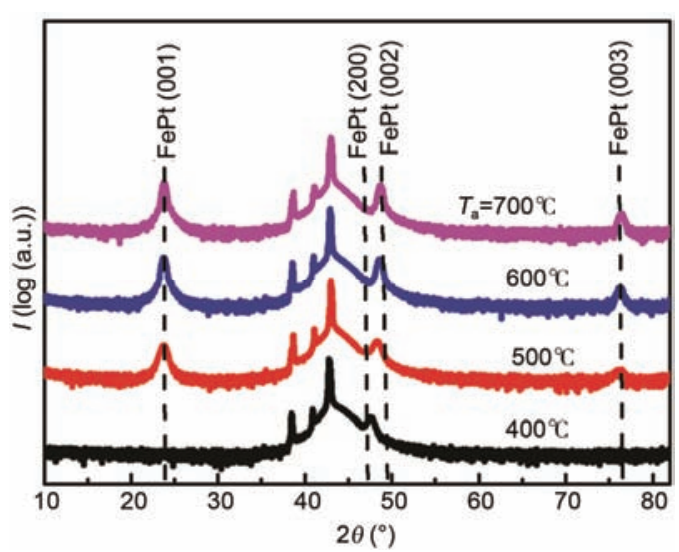

图 1 (网络版彩色) $\mathrm{FePt}(30 \mathrm{~nm}$ )薄膜在不同温度进行热处理后的 $\mathrm{XRD}$ 图谱. 图中只标出了 $\mathrm{FePt}$ 薄膜的织构峰, 其他峰均来自 $\mathrm{MgO}$ (001)基片

Figue 1 (Color online) XRD spectra for annealed $\mathrm{FePt}(30 \mathrm{~nm})$ films. Indexed peaks were from (001)-textured FePt

右移，应该是薄膜沿面内方向受到基片的拉伸应力 (但没有发生 $A 1 \rightarrow L 1_{0}$ 相变) 的结果, 即晶格畸变导致 垂直于膜面方向的 $c$ 轴缩短. $T_{\mathrm{a}}=500{ }^{\circ} \mathrm{C}$, 开始出现 (001)和(003)峰, 表明薄膜内开始形成 $L 1_{0}$ 相, 但强度 较弱, 由此断定薄膜中 $L 1_{0}$ 相和 $A 1$ 相共存. $L 1_{0}$ 相的 $c$ 轴更短, 所以 (200)峰继续右移. 此时的(200)峰应该 是两相分别形成衍射峰的叠加. 为了区别, 把属于 $A 1$ 相的衍射峰表示为 $(h 00)$, 而把属于 $L 1_{0}$ 相的表示为 $(00 l) . T_{\mathrm{a}}=600^{\circ} \mathrm{C},(001)$ 峰和(003)峰更加明显, 说明有 更多的 $A 1$ 相转变成了 $L 1_{0}$ 相, 基础峰更靠近 $(002)$ 峰的 位置. $T_{\mathrm{a}}=700^{\circ} \mathrm{C}$, 基础峰与 (002)峰的位置基本重合, 薄膜内 $A 1 \rightarrow L 1_{0}$ 转变接近完成. 这样, 通过改变热处 理温度, 就得到 $A 1 \rightarrow L 1_{0}$ 转变程度不同的 FePt薄膜.

表征 $A 1 \rightarrow L 1_{0}$ 转变程度的物理量是有序度 $S$, 范 围在 $0 \sim 1$ 之间, 越接近 1 , 有序化程度越高. $S$ 可以通 过XRD数据计算得出 ${ }^{[27,28]}$.

$$
S^{2}=\left(I_{001} / I_{002}\right)_{\exp } /\left(I_{001} / I_{002}\right)_{\text {cal }},
$$

式中 $\left(I_{001} / I_{002}\right)_{\exp }$ 为 $\mathrm{FePt}(001)$ 超晶格峰与 $(002)$ 基础峰 的XRD观测强度比值, $I_{002}$ 是(200)峰和(002)峰积分强 度的总和. $\left(I_{001} / I_{002}\right)_{\mathrm{cal}}$ 是理论计算强度比. 计算要考 虑 $\mathrm{X}$ 射线强度随穿人深度的衰减与 Debye-Waller因 子. 对 XRD数据进行处理得到, $T_{\mathrm{a}}=500,600,700^{\circ} \mathrm{C}$ 对 应的 $S$ 分别为 $0.61,0.84$ 和 0.90 .

图 2 为 $F e P t(30 \mathrm{~nm})$ 薄膜在不同温度进行热处理的 AFM图像. $T_{\mathrm{a}}=400^{\circ} \mathrm{C}$ (图 2(a)), 薄膜由细小均匀的晶 粒组成, 均匀地覆盖在基片上, 覆盖率为 $100 \%$, 表
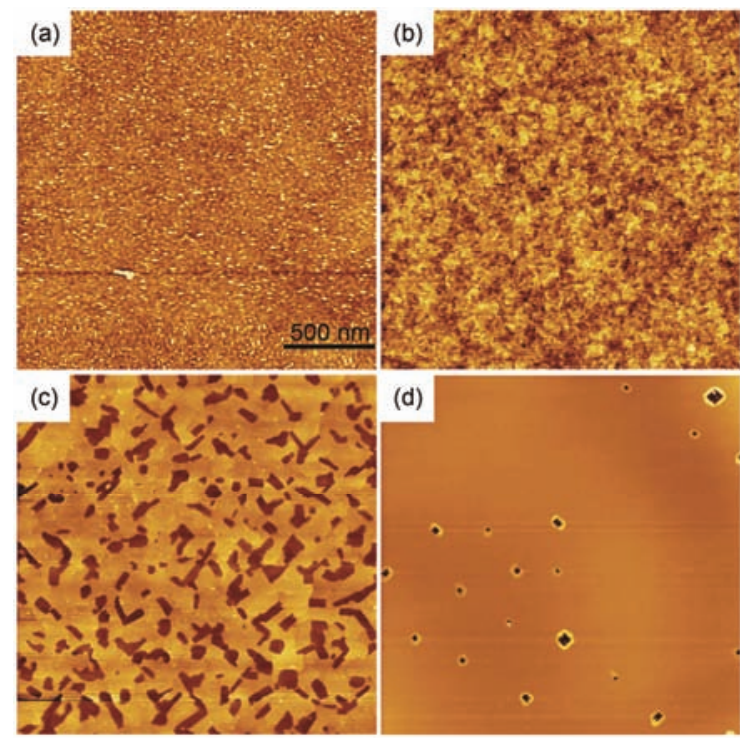

(d)

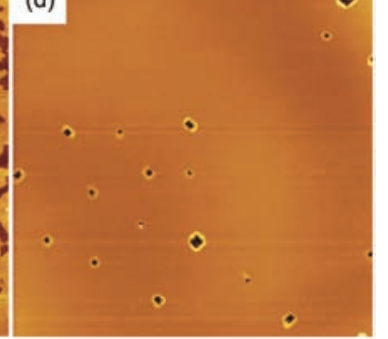

图 2 (网络版彩色) $\mathrm{FePt}\left(30 \mathrm{~nm}, T_{\mathrm{a}}\right.$ ) 薄膜的 AFM图像. $T_{\mathrm{a}}=400$ (a), 500 (b), 600 (c), $700^{\circ} \mathrm{C}$ (d)

Figure 2 (Color online) AFM images for annealed $\mathrm{FePt}\left(30 \mathrm{~nm}, T_{\mathrm{a}}\right)$ films. $T_{\mathrm{a}}=400(\mathrm{a}), 500(\mathrm{~b}), 600(\mathrm{c}), 700^{\circ} \mathrm{C}(\mathrm{d})$

面最大高低差为 $2.45 \mathrm{~nm}$ (根据明暗强度确定, 图中 未给出标度尺). $T_{\mathrm{a}}=500^{\circ} \mathrm{C}$ (图2(b)), 在晶格应力和热 振动的共同作用下，薄膜内应力大或温度高的区域 开始有原子向自由能更低的位置跳跃, 原子层平铺 延展, 晶粒变平, 尺寸变大, 间距变窄, 接触的晶粒 相互合并. 原子层不完整的位置出现台阶，形成浅 坑. 但是没有露出基底, 覆盖率仍然是 100\%, 表面 最大高低差仅有 $1.02 \mathrm{~nm} . T_{\mathrm{a}}=600^{\circ} \mathrm{C}$ (图2(c)), 原子层 完整的部分相互连通, 呈现出网状结构, 原子台阶围 成网孔, 孔内有若干原子层中断, 形成较深的平底 坑, 坑的边缘多呈直线, 反映晶体外形的规则性. X 射线能谱分析表明, 坑内存在Fe和Pt两种元素, 说明 仍未露出基底, 覆盖率还是 $100 \%$. 膜面最大高低差 为 $4.77 \mathrm{~nm} . T_{\mathrm{a}}=700^{\circ} \mathrm{C}$ (图 2(d)), 薄膜局部的连续性被 破坏, 出现边缘隆起的方孔. 最大高低差增大到 49 $\mathrm{nm}$, 表明孔内已经露出基片 (最大高低差超过膜厚, 是孔边缘隆起导致). 受方孔边缘隆起影响, 难以分 辨方孔以外平整区域的高度差, 但实际上已经裂成 孤立的岛状晶片. 这通过测量磁化曲线(图3(d))得到 证实.

图3为 FePt $(30 \mathrm{~nm})$ 薄膜在不同温度进行热处理后 的磁化曲线. 图中[001]和 [100]曲线的磁场分别沿垂 直和平行于膜面的方向施加.

$T_{\mathrm{a}}=400^{\circ} \mathrm{C}$ (图3(a)), 由于 $\mathrm{FePt}$ 薄膜呈软磁性, 磁 

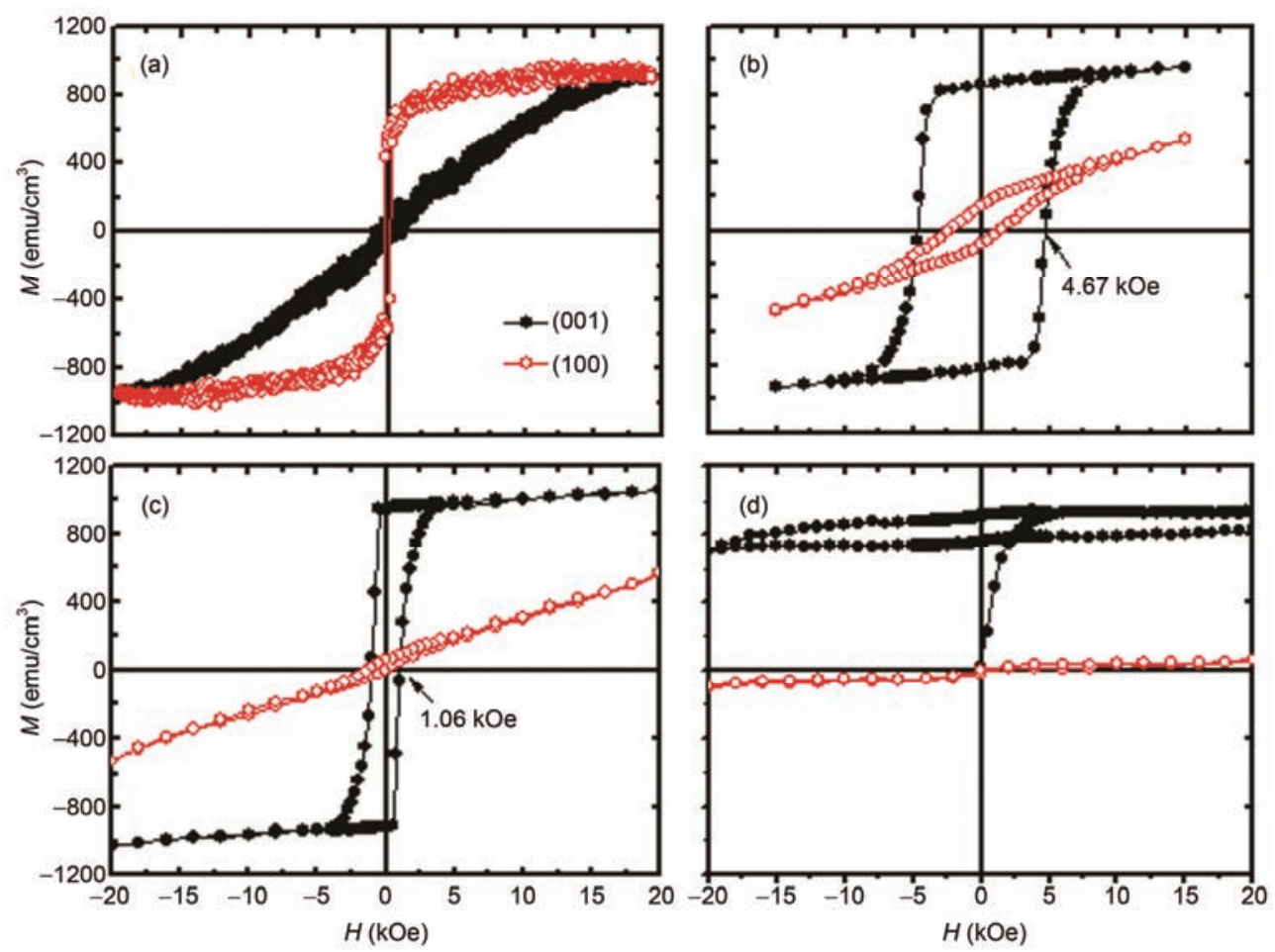

图 3 (网络版彩色) FePt $\left(30 \mathrm{~nm}, T_{\mathrm{a}}\right.$ 薄膜的 $M-H$ 曲线. $T_{\mathrm{a}}=400(\mathrm{a}), 500(\mathrm{~b}), 600(\mathrm{c}), 700^{\circ} \mathrm{C}$ (d), 实心和空心点分别表示外磁场沿垂直和平行于膜面 的方向施加

Figure 3 (Color online) Magnetization curves for annealed FePt (30 nm, $T_{\mathrm{a}}$ ) films. $T_{\mathrm{a}}=400$ (a), 500 (b), 600 (c), $700^{\circ} \mathrm{C}$ (d). Solid and hollowed dots represent magnetic fields were applied along out-of-plane and in-plane directions, respectively

化行为主要由形状各向异性决定，所以易磁化轴明 显位于膜面内, 矫顽力很小. 沿垂直于膜面方向磁 化, 磁化曲线几乎为一条可逆的直线, 是磁矩在外场 作用下抵抗退磁场, 逐渐由易轴方向 (面内)转向难轴 方向(垂直)的结果.

$T_{\mathrm{a}}=500^{\circ} \mathrm{C}$ (图3(b)), 虽然 $\mathrm{FePt}$ 薄膜由 $A 1$ 和 $L 1_{0}$ 两相 混合而成, 但易磁化轴已经明显地切换到与膜面垂 直的方向. [001]曲线呈矩形, 没有看到软磁相单独反 转的过程, $H_{\mathrm{c}}=4.67 \mathrm{kOe}$. 这说明尽管有序化程度还不 高 $(S=0.61)$, 但薄膜自身已经形成了良好的 $L 1_{0} / A 1-$ $\mathrm{FePt}$ 复合体, 两相间存在强烈的交换耦合作用. 在垂 直于膜面方向, 被钉扎的软磁相只能随着硬磁相一 起发生磁化反转, 表现为刚性的硬磁行为. 而[100] 曲线的磁化强度变化幅度要小得多, 是因为 $L 1_{0}$ 相的 磁矩在外场作用下只能小幅偏离垂直方向向膜面方 向靠拢, 不形成磁畴. 磁矩偏离的角度不大, 所以对 磁化强度贡献较小. 但回线还是有一定的面积, 是因 为在面内磁场和形状各向异性场的共同作用下, 软 磁相的部分磁矩能够转向面内方向, 但受到周围硬
磁相的制约, 转动不可逆 ${ }^{[23]}$.

$T_{\mathrm{a}}=600^{\circ} \mathrm{C}$ (图3(c)), 薄膜内 $A 1$ 相进一步减少, 但 是 [001] 曲线的 $H_{\mathrm{c}}$ 反而下降到 $1.06 \mathrm{kOe}$, 磁化反转阶 段的曲线变化更陡峻. 这是因为薄膜内小晶粒相互 合并, 形成了面积大的连续晶片(图2(c)), 一旦晶片 内部形核, 反向磁畴的畴壁就能够在相当大的范围 内自由移动, 迅速引起大片薄膜的磁矩反转. [100]曲 线则几乎是一条直线, 但回线仍然在低磁场阶段有 面积, 表明薄膜内确实还存在软磁相 $(S=0.84)$.

$T_{\mathrm{a}}=700{ }^{\circ} \mathrm{C}$ (图3(d)), [001]曲线初始段的磁化强度 从零开始快速增加, 在 $H=5 \mathrm{kOe}$ 以内就趋于饱和. 这 表明薄膜内多数孤立晶片的尺寸大于单磁畴临界尺 寸, 热消磁状态形成了多个磁畴, 施加外场后, 依靠 畴壁移动比较容易被磁化. 但施加 $20 \mathrm{kOe}$ 的磁场后, 再反复施加磁场, 磁化强度却不再大幅变化. 这是由 于孤立的晶片一旦被磁化到饱和, 变成单个磁畴, 就 只能依靠形核才能发生磁化反转. 而形核场很大, 高 于设备提供的最大磁场, 因此未能测出 $H_{\mathrm{c}}$. [100]曲线 则几乎是一条过原点的水平直线, 说明薄膜已经基 
本转变为磁晶各向异性能大的 $L 1_{0}$ 相 $(S=0.90$, 没有很 接近 1 , 可能是计算方法不够精确, 但也不排除残存 $A 1$ 相尺度已经很小的可能性, 造成 $S$ 未达到理想值, 但薄膜的磁性已经与纯 $L 1_{0}$ 相的相当接近). 有关这种 高有序度 FePt硬磁薄膜在更强磁场中的磁化反转机 制，可以参考文献[21].

根据图 1 3 的结果, 在 $400^{\circ} \mathrm{C}$ 进行热处理, 薄膜 没有发生有序化; 而在 $700^{\circ} \mathrm{C}$ 进行热处理, 薄膜的连 续性开始被破坏且 $H_{\mathrm{c}}$ 非常大. 所以制备硬磁/软磁磁 性弹簧, 应该主要使用 $T_{\mathrm{a}}=500$ 和 $600^{\circ} \mathrm{C}$ 两种硬磁层.

\section{$2.2 \quad L 1_{0}-\operatorname{FePt}\left(30 \mathrm{~nm}, T_{\mathrm{a}}\right) / A 1-\mathrm{FePt}(x \mathrm{~nm})$ 双层薄膜 的性质}

$T_{\mathrm{a}}=500$ 和 $600^{\circ} \mathrm{C}$ 两种硬磁层内也包含交换弹性作 用. 但是无法对这种层内作用进行定量分析. 做成硬 磁/软磁双层膜, 人为控制两层的生长条件和厚度, 可以方便地分析层间交换作用. 硬磁层和软磁层之 间的交换耦合有一定的作用范围. 软磁层自身的矫 顽力很小, 磁化方向容易受到外场和形状各向异性 的影响. 而硬磁层矫顽力大, 改变磁化方向需要很强 的外场. 形成复合双层膜, 如果软磁层很薄, 则所有 的软磁物质都受到硬磁层交换耦合作用制约, 磁矩 只能随硬磁层在外场中一起反转, 表现为刚性行为, 磁化曲线上只看到单一开关场 ${ }^{[14]}$. 增加软磁层的厚 度, 远离界面的软磁物质受到硬磁层的钉扎作用减 弱, 从垂直于界面的方向看, 软磁层内的磁矩逐渐改 变旋转角度, 离界面越远, 磁矩越倾向于指向外场方 向. 如果反向磁场小于硬磁层的反转场, 则软磁层内 的磁矩旋转是可逆的. 外场消失, 软磁层的磁矩又回 到与硬磁层磁矩平行的方向, 这就是磁性弹簧现 象 ${ }^{[29 ~ 31]}$. 软磁层厚度超过某一数值, 远离界面的软 磁物质就可以单独形核产生反向磁畴. 反向磁畴扩 大, 畴壁被磁场压向界面. 磁场越强, 畴壁离界面越 近, 直到突破界面引起硬磁层的磁矩突然反转. 反磁 化曲线上会出现两个磁化强度明显下降的拐点, 称 为双肩现象 ${ }^{[5,13,32]}$. 出现双肩现象的软磁层临界厚度 $\left(l_{\mathrm{ex}}\right)$ 就反映硬磁/软磁界面两侧物质的交换作用长度. 把握了层间交换作用长度, 可以分析决定交换弹性 作用强弱的关键因素, 从而认识磁化反转机制, 并主 动向有利于应用的方向对性质进行调控.

$L 1_{0}-\mathrm{FePt}$ 的布洛赫型畴壁厚度为 ${ }^{[33]}$

$$
w_{\mathrm{dw}}=\pi\left(A / K_{\mathrm{u}}\right)^{1 / 2},
$$

式中交换作用常数 $A=10^{-6} \mathrm{erg} / \mathrm{cm}$ ，由材料的自旋量 子数和晶格常数共同决定. 按照磁性弹簧理论, $l_{\mathrm{ex}}=2 w_{\mathrm{dw}}$, 即软磁层的厚度超过硬磁层畴壁厚度的 2 倍, 磁化曲线出现双肩现象 ${ }^{[14,31]}$.

图4是 $L 1_{0}-\mathrm{FePt}(001)\left(30 \mathrm{~nm}, T_{\mathrm{a}}=500^{\circ} \mathrm{C}\right) / A 1-\mathrm{FePt}(x$ $\mathrm{nm}$ ) 双层薄膜的 $M-H$ 曲线. 只有硬磁层, 即 $x=0$ 时 (图 $4(\mathrm{a}))$, 内部 $L 1_{0}$ 相和 $A 1$ 相共存, 两相间形成交换作用 耦合体, 沿易轴方向磁化的磁化曲线呈方形, $H_{\mathrm{c}}=$ $4.67 \mathrm{kOe}$. 覆盖上 $20 \mathrm{~nm}$ 厚的软磁层(图4(b)), 磁化曲 线仍然保持为方形, $H_{\mathrm{c}}$ 也没有明显变化, 双层膜表现 为刚性行为, 说明软磁层的厚度小于界面交换作用 长度. 软磁层增加到 $30 \mathrm{~nm}$ (图4(c)), 磁化曲线不再是 方形, $H_{\mathrm{c}}$ 下降到 $4.02 \mathrm{kOe}$. 在 $H=H_{1}$ 和 $H_{2}$ 两个特征磁场 附近, 磁化强度突然变化 (即出现了双肩现象). $H_{1}$ 为 软磁层的形核场, $H_{2}$ 为硬磁层的不可逆反转场. 在 $H_{1}$ 和 $\mathrm{H}_{2}$ 之间是软磁层反转的过程, 但磁化强度并没有 在 $H_{1}$ 突然下降然后形成平台, 是形状各向异性造成 的. 在垂直于膜面方向, 退磁因子最大 $(N \approx 1)$, 对软 磁层来说是难轴方向, 所以不受硬磁层钉扎的软磁 层磁矩倾向于逐渐转向外磁场方向, 而不是依靠畴 壁移动. 从[100]曲线看, 图4(c)的磁化强度在磁场为 零附近有突然变化, 说明软磁层的磁矩能够自由反 转, 因为沿此方向磁化的退磁因子最小 $(N \approx 0)$, 是软 磁层的易轴方向. 而图 $4(\mathrm{~b})$ 的软磁层还不够厚, 磁矩 要受到硬磁层很强的约束, 转向面内方向要困难得 多. 软磁层与硬磁层中的软磁相不同, 没有被硬磁相 包围, 所以沿[100]方向磁化, 不会发生不可逆反转. 因此, 图4(b)和(c)中 [100]回线的面积应该主要是由 硬磁层中的软磁相贡献的, 与软磁层基本无关. 总 之, 根据图 $4, T_{\mathrm{a}}=500{ }^{\circ} \mathrm{C}$ 的层间交换作用长度应该在 $20 \mathrm{~nm}<l_{\mathrm{ex}}<30 \mathrm{~nm}$ 范围.

图5是 $L 1_{0}-\mathrm{FePt}(001)\left(30 \mathrm{~nm}, T_{\mathrm{a}}=600^{\circ} \mathrm{C}\right) / A 1-\mathrm{FePt}(x$ $\mathrm{nm})$ 双层薄膜的 $M-H$ 曲线. 与图 4 相比, 硬磁层中 $A 1$ $\rightarrow L 1_{0}$ 转变程度加深, 但仍有 $A 1$ 相残留 (因为图 5(a) 的 [100]曲线还有面积). 由于 $L 1_{0}$ 相已经连成大片的 连续晶体, 沿易轴方向磁化的 $H_{\mathrm{c}}$ 下降到 $1.06 \mathrm{kOe}$. 当 $x=20 \mathrm{~nm}$ (图5(b)), $H_{\mathrm{c}}$ 没有明显变化, 磁化曲线仍然表 现为刚性行为. [100]曲线与图 5(a)相似, 表明软磁层 的磁矩同样在层间交换作用下克服了形状各向异性, 指向与硬磁层磁矩平行的方向. 当 $x=30 \mathrm{~nm}$ (图5(c)), 和图4(c)一样, 也出现双肩现象. [100]曲线同样在磁 场为零附近突然变化, 也是软磁层中远离硬磁层位 


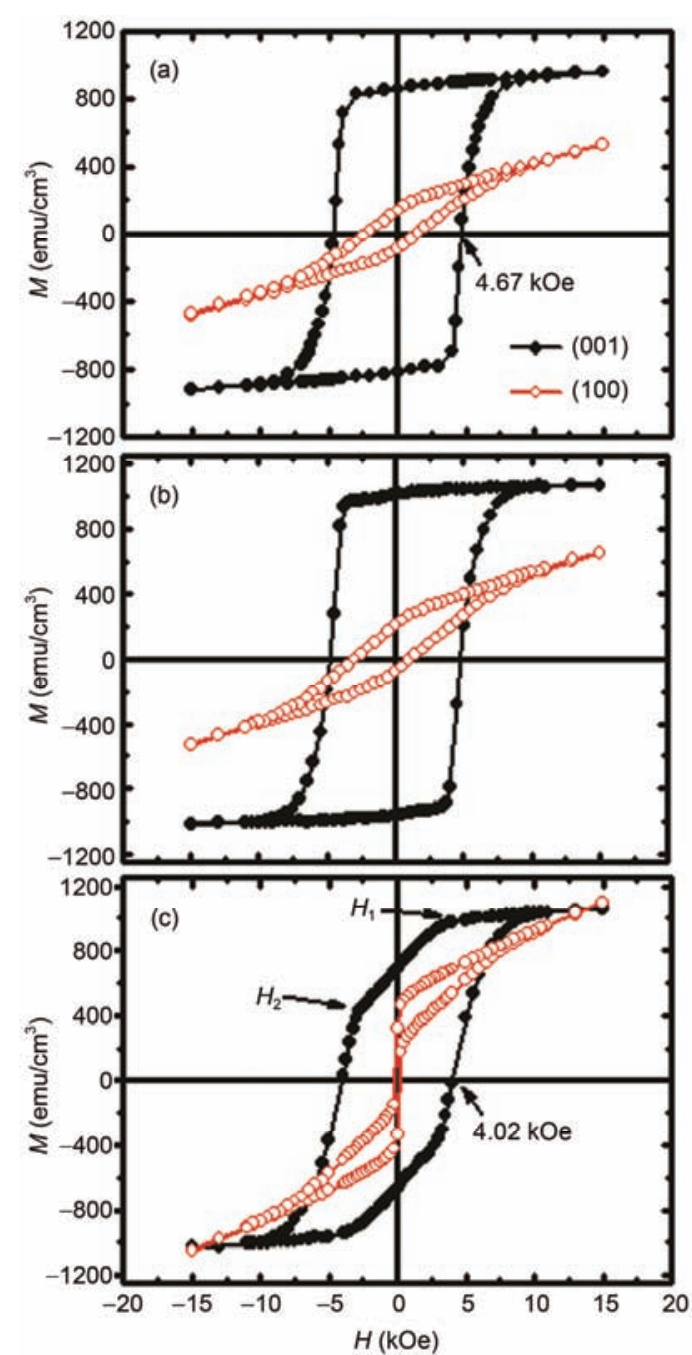

图 4 (网络版彩色) $L 1_{0}-\mathrm{FePt}\left(30 \mathrm{~nm}, 500^{\circ} \mathrm{C}\right) / A 1-\mathrm{FePt}(x \mathrm{~nm})$ 双层薄膜 $M-H$ 曲线, $x=0$ (a), 20 (b), 30 (c)

Figure 4 (Color online) Magnetization curves for $L 1_{0}-\mathrm{FePt}(30 \mathrm{~nm}$, $\left.500^{\circ} \mathrm{C}\right) / A 1-\mathrm{FePt}(x \mathrm{~nm})$ bilayer films, $x=0$ (a), 20 (b), 30 (c)

置的磁矩脱离了硬磁层的钉扎作用, 在形状各向异 性的作用下，倾向于指向面内方向造成的. 所以, $T_{\mathrm{a}}=$ $600^{\circ} \mathrm{C}$ 的层间交换作用长度仍然满足 $20 \mathrm{~nm}<l_{\mathrm{ex}}<30 \mathrm{~nm}$.

对于理想的 $L 1_{0}-\mathrm{FePt}$, 由(2)式得到, $w_{\mathrm{dw}}=\sim 5$ $\mathrm{nm}^{[34]}$. 因此 $l_{\mathrm{ex}} \approx 10 \mathrm{~nm}$. 但是根据图 4 和 $5, l_{\mathrm{ex}}>20 \mathrm{~nm}$. 这说明 (2)式中的 $A / K_{\mathrm{u}}$ 不会低于理想值的 4 倍. $A=$ $A_{\mathrm{ex}} S^{2}{ }_{\text {spin }} / a^{[35]}$. 对于同一种物质, 交换劲度常数 $A_{\mathrm{ex}}$ 和 自旋量子数 $S_{\mathrm{spin}}$ 不会有多大变化, 只有晶格常数 $a$ 确 实和热处理温度有关. 然而, $A 1 \rightarrow L 1_{0}$ 相变引起的 $a, b$ 变长和 $c$ 变短, 不可能导致 $A$ 发生能使 $l_{\mathrm{ex}}$ 倍增的结果. 这可以通过对比图 4 和 5 进行验证. $T_{\mathrm{a}}=600^{\circ} \mathrm{C}$, 硬磁层 内 $L 1_{0}-\mathrm{FePt}$ 占据优势, 残存的 $A 1-\mathrm{FePt}$ 少, 晶格常数已

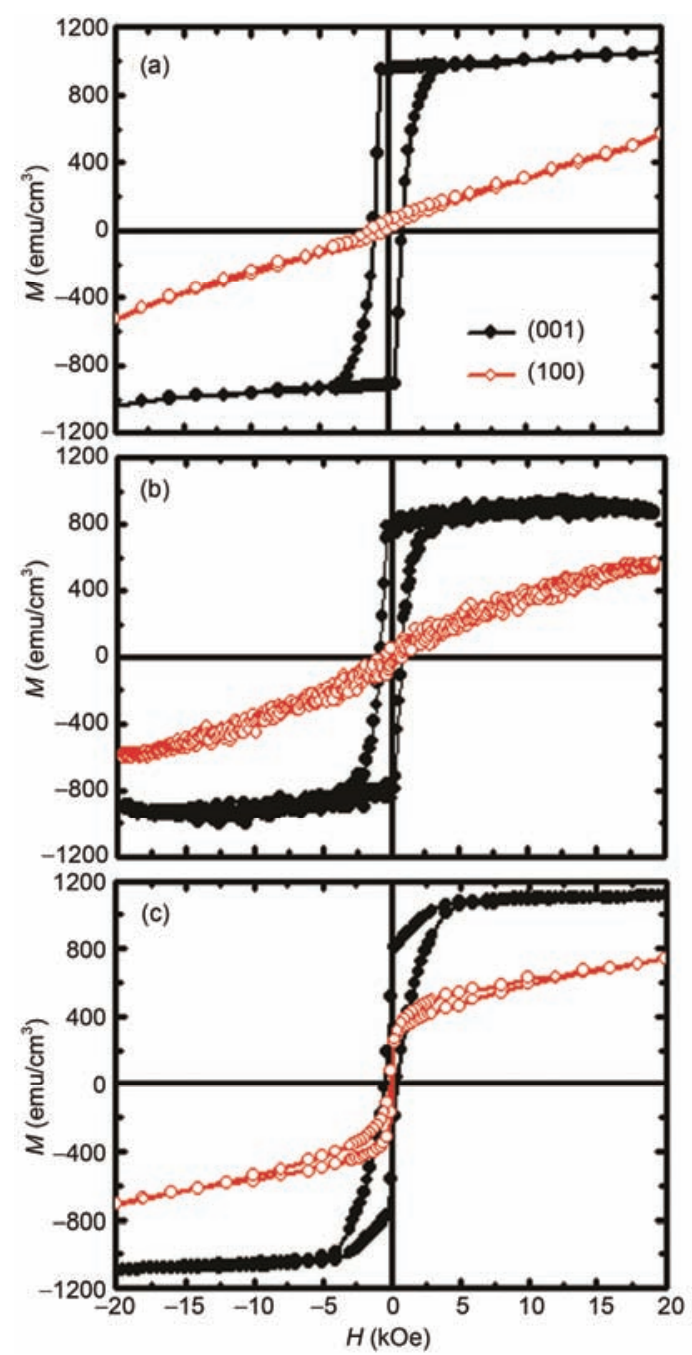

图 5 (网络版彩色) $L 1_{0}-\mathrm{FePt}\left(30 \mathrm{~nm}, 600^{\circ} \mathrm{C}\right) / A 1-\mathrm{FePt}(x \mathrm{~nm})$ 双层薄膜 $M-H$ 曲线, $x=0$ (a), 20 (b), 30 (c)

Figure 5 (Color online) Magnetization curves for $L 1_{0}-\mathrm{FePt}(30 \mathrm{~nm}$, $\left.600^{\circ} \mathrm{C}\right) / A 1-\mathrm{FePt}(x \mathrm{~nm})$ bilayer films, $x=0$ (a), 20 (b), 30 (c)

经与理想 $L 1_{0}-\mathrm{FePt}$ 的接近. 但是实验确定的 $l_{\mathrm{ex}}$ 范围与 $T_{\mathrm{a}}=500{ }^{\circ} \mathrm{C}$ 的情况一样. 因此, $A$ 产生差异不是 $l_{\mathrm{ex}}$ 发生 变化的关键原因.

排除了 $A$, 根据(2)式，影响 $l_{\mathrm{ex}}$ 最明显的因素就只 剩下 $K_{\mathrm{u}}$. 由于硬磁层并没有完全有序化, 有效磁晶各 向异性能与理想 $L 1_{0}-\mathrm{FePt}$ 合金相比会低得多，因此可 以大幅增大层间交换作用长度. 与 $T_{\mathrm{a}}=500{ }^{\circ} \mathrm{C}$ 相比, $T_{\mathrm{a}}=600^{\circ} \mathrm{C}$ 的 $l_{\mathrm{ex}}$ 并没有明显缩短, 说明只要硬磁层内 残存足够的 $A 1$ 相，就会导致 $K_{\mathrm{u}}$ 大幅下降. 找到了决 定 $l_{\mathrm{ex}}$ 的主要因素, 就可以根据需要来设计磁性弹簧 的表现. 这也说明，至少在 $0.61<S<0.84$ 范围, $l_{\mathrm{ex}}$ 都不 短于 $20 \mathrm{~nm}$, 也不长于 $30 \mathrm{~nm}$, 为热处理硬磁层提供 
了较大的温度灵活性, 从而可以更多地关注热处理 对表面高低差(厚度的均匀性)的影响.

图 6 是 $L 1_{0}-\mathrm{FePt}(001)\left(30 \mathrm{~nm}, T_{\mathrm{a}}=700{ }^{\circ} \mathrm{C}\right) / A 1-\mathrm{FePt}$ $(20 \mathrm{~nm})$ 双层薄膜的 $M-H$ 曲线. 与图4(b)和 5(b)不同的 是, [001]曲线观察到了软磁层磁矩的单独反转. 而硬 磁层却和图3(d)一样, 一旦被磁化到饱和, 在设备能 提供的磁场范围内不发生磁化反转. 这表明 $l_{\mathrm{ex}}<20$ $\mathrm{nm}$, 明显是 $K_{\mathrm{u}}$ 增大导致. 这有效验证了以上关于 $l_{\mathrm{ex}}$ 决定因素的讨论. 需要注意的是, 图6中软磁层没有 与硬磁层一起表现为刚性行为, [100]曲线却没有像 图4(c)和5(c)那样, 在磁场为零附近出现磁化强度的 突然变化. 这说明厚度为 $20 \mathrm{~nm}$ 的软磁层内虽然可以 单独形核, 但在离硬磁层最远的地方, 磁矩也不是自 由的，而是受硬磁层影响，仍然能够克服形状各向异 性，指向垂直于膜面的方向.

\section{3 结论}

在加热到 $400^{\circ} \mathrm{C}$ 的 $\mathrm{MgO}(001)$ 单晶基片上, 用磁控 溅射法取向生长 $30 \mathrm{~nm}$ 厚的 $\mathrm{FePt}$ 薄膜, 在不同温度进 行热处理, 使其发生不同程度的 $A 1 \rightarrow L 1_{0}$ 转变. 然后 在 $100^{\circ} \mathrm{C}$ 生长厚度为 20 和 $30 \mathrm{~nm}$ 的第二层 $\mathrm{FePt}$, 得到 沿[001]方向取向生长的 $L 1_{0}-\mathrm{FePt}\left(30 \mathrm{~nm}, T_{\mathrm{a}}\right) / A 1-\mathrm{FePt}$ $(x \mathrm{~nm})$ 同材异质双层薄膜, 对这种硬磁/软磁交换耦 合体系的层间交换弹性作用进行了分析. 热处理温 度 $T_{\mathrm{a}}$ 为 500 和 $600^{\circ} \mathrm{C}$, 硬磁层都能够保持 $100 \%$ 的覆盖 率, 但是前者的矫顽力要大得多(接近 $5 \mathrm{kOe}$ ), 是晶 粒尺寸不一样造成的. 尽管硬磁层内 $L 1_{0}$ 相与 $A 1$ 相

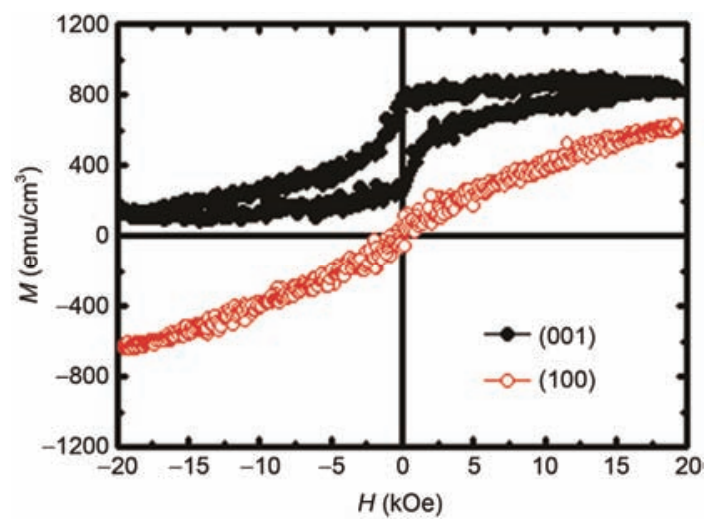

图 6 (网络版彩色) $L 1_{0}-\mathrm{FePt}\left(30 \mathrm{~nm}, 700^{\circ} \mathrm{C}\right) / A 1-\mathrm{FePt}(20 \mathrm{~nm})$ 双层薄膜 $M-H$ 曲线

Figure 6 (Color online) Magnetization curves for $L 1_{0}-\mathrm{FePt}(30 \mathrm{~nm}$ $\left.700^{\circ} \mathrm{C}\right) / A 1-\mathrm{FePt}(20 \mathrm{~nm})$ bilayer film

共存，但表现出垂直磁晶各向异性，磁化曲线都呈方 形. 软磁层厚度为 $x=20 \mathrm{~nm}$, 双层薄膜的磁化曲线仍 然保持方形形状，软磁层和硬磁层的磁矩一起反转, 表现为刚性行为. 而 $x=30 \mathrm{~nm}$, 磁化曲线上出现双肩 现象, 软磁层和硬磁层分两次各自反转. 与理想的 $L 1_{0} / A 1-\mathrm{FePt}$ 交换耦合体系相比，层间交换作用长度 增大了 $100 \%$ 以上, 是硬磁层内 $L 1_{0}$ 相与 $A 1$ 相共存, 降 低了有效单轴磁晶各向异性引起的. $T_{\mathrm{a}}=700^{\circ} \mathrm{C}$, 则 $x=20 \mathrm{~nm}$ 就可以观察到软磁层磁矩单独反转. 对硬磁 层在 $500,600,700^{\circ} \mathrm{C}$ 进行热处理，有序度分别为 0.61 , 0.84和 0.90 . 通过改变硬磁层的 $A 1 \rightarrow L 1_{0}$ 转变程度, 可 以同时调控矫顽力和层间交换弹性作用长度，为不 同应用背景设计各种磁性弹簧提供了方便.

\section{参考文献}

1 Suess D. Micromagnetics of exchange spring media: Optimization and limits. J Magn Magn Mater, 2007, 308: 183-197

2 Rong C B, Nandwana V, Poudyal N, et al. Bulk FePt-based nanocomposite magnets with enhanced exchange coupling. J Appl Phys, 2007, 102: 023908-023914

3 Weller D, Moser A, Folks L, et al. High $K_{\mathrm{u}}$ materials approach to $100 \mathrm{Gbits} / \mathrm{in}^{2}$. IEEE Trans Magn, 2000, 36: 10-15

4 Skomski R. Nanomagnetics. J Phys-Condens Matter, 2003, 15: R841-R896

5 Goll D, Bublat T. Large-area hard magnetic $L 1_{0}$-FePt and composite $L 1_{0}$-FePt based nanopatterns. Phys Status Solid A, 2013, 210: $1261-1271$

6 Ishihara S, Ohtake M, Futamoto M. Magnetic force microscope tips coated with FePd, FePt, and CoPt alloy films. Thin Solid Films, 2013, 546: 205-210

7 Weller D, Moser A. Thermal effect limits in ultrahigh-density magnetic recording. IEEE Trans Magn, 1999, 35: 4423-4439

8 Sun S H. Recent advances in chemical synthesis, self-assembly, and applications of FePt nanoparticles. Adv Mater, 2006, 18: 393-403

9 Weller D, McDaniel T. Media for Extremely High Density Recording. Advanced Magnetic Nanostructures. New York: Springer, 2006. 295-324

10 Suzuki T, Honda N, Ouchi K. Magnetization reversal process in polycrystalline ordered Fe-Pt(001) thin films. J Appl Phys, 1999, 85: 4301-4303

11 Coffey K R, Parker M A, Howard J K. High anisotropy $L 1_{0}$ thin films for longitudinal recording. IEEE Trans Magn, 1995, 31: 2737-2739 
12 Sun S H, Murray C B, Weller D, et al. Monodisperse FePt nanoparticles and ferromagnetic FePt nanocrystal superlattices. Science, 2000, 287: 1989-1992

13 Shima T, Takanashi K, Takahashi Y K, et al. Nucleation-type magnetization behavior in FePt(001) particulate films. J Appl Phys, 2006, 99: 033516

14 Kneller E F, Hawig R. The exchange-spring magnet: A new material principle for permanent magnets. IEEE Trans Magn, 1991, 27: $3588-3560$

15 Yan S S, Liu W J, Weston J L, et al. Magnetization-reversal mechanism of hard/soft exchange-coupled trilayers. Phys Rev B, 2001, 63: 174415

16 Mangin S, Marchal G, Bellouard C, et al. Magnetic behavior and resistivity of the domain-wall junction $\mathrm{GdFe}(1000 \AA) / \mathrm{TbFe} / \mathrm{GdFe}(500$ Å). Phys Rev B, 1998, 58: 2748

17 Tsai J L, Tzeng H T, Liu B F. Coercivity variation in exchange-coupled Fe/FePt bilayer with perpendicular magnetization. Thin Solid Films, 2010, 518: 7271-7274

18 Wang J S, Yang B, Pei W L, et al. Structural and magnetic properties of $L 1_{0}-\mathrm{FePt} / \mathrm{Fe}$ exchange coupled nano-composite thin films with high energy product. J Magn Magn Mater, 2013, 345: 165-170

19 Casoli F, Albertini F, Nasi L, et al. Strong coercivity reduction in perpendicular FePt/Fe bilayers due to hard/soft coupling. Appl Phys Lett, 2008, 92: 142506

20 Davies J E, Hellwig O, Fullerton E E, et al. Anisotropy dependence of irreversible switching in Fe/SmCo and FeNi/FePt exchange spring magnet films. Appl Phys Lett, 2005, 86: 262503

21 Shima T, Takanashi K, Takahashi Y K, et al. High coercivity and magnetic domain observation in epitaxially grown particulate FePt thin films. J Magn Magn Mater, 2003, 266: 171-177

22 Zhang Y, Wan J, Skumryev V, et al. Microstructural characterization of $L 1_{0} \mathrm{FePt} / \mathrm{MgO}$ nanoparticles with perpendicular anisotropy. Appl Phys Lett, 2004, 85: 5343-5345

23 Li G Q, Zhu Y Y, Zhang Y, et al. Exchange spring in $A 1 / L 1_{0}$ FePt composite and its application in magnetic force microscope. Appl Phys Lett, 2015, 106: 082404

24 Ristau R A, Barmak K, Lewis L H, et al. On the relationship of high coercivity and $L 1_{0}$ ordered phase in CoPt and FePt thin films. J Appl Phys, 1999, 86: 4527-4533

25 Takahashi Y, Matsubara E, Kawazoe Y, et al. Reconstruction of atomic images from multiple-energy X-ray holograms of FePt films by the scattering pattern matrix method. Appl Phys Lett, 2005, 87: 234104

26 Shima T, Moriguchi T, Seki T, et al. Fabrication of $L 1_{0}$ ordered FePt alloy films by monatomic layer sputter deposition. J Appl Phys, 2003, 93: 7238-7240

27 Okamoto S, Kitakami O, Shimada Y. Crystal distortion and the magnetic moment of epitaxially grown $\alpha^{\prime \prime}-\mathrm{Fe}_{16} \mathrm{~N}_{2}$. J Magn Magn Mater, 2000, 208: 102-114

28 Thiele J U, Folks L, Toney M F, et al. Perpendicular magnetic anisotropy and magnetic domain structure in sputtered epitaxial FePt( 001$)$ $L 1_{0}$ films. J Appl Phys, 1998, 84: 5686-5692

29 Schrefl T, Forster H, Dittrich R, et al. Reversible magnetization processes and energy density product in $\mathrm{Sm}-\mathrm{CoFe}$ and $\mathrm{Sm}-\mathrm{Co} / \mathrm{Co}$ bilayers. J Appl Phys, 2003, 93: 6489-6491

30 Jiang J S, Bader S D, Kaper H, et al. Rotational hysteresis of exchange-spring magnets. J Phys D-Appl Phys, 2002, 35: 2339-2343

31 Jiang J S, Bader S D. Magnetic reversal in thin film exchange-spring magnets. Scr Mater, 2002, 47: 563-568

32 Liu J P, Luo C P, Liu Y, et al. High energy products in rapidly annealed nanoscale Fe/Pt multilayers. Appl Phys Lett, 1998, 72: 483-485

33 Song Y, Zhang Z, Duan N, et al. Composition and size dependence of magnetic properties of FePt/Fe exchange-spring films. J Magn Magn Mater, 2014, 371: 100-105

34 Li G Q, Takahoshi H, Ito H, et al. Morphology and domain pattern of $L 1_{0}$ ordered FePt films. J Appl phys, 2003, 94: 5672-5677

35 Klemmer T, Hoydick D, Okumura H, et al. Magnetic hardening and coercivity mechanisms in $L 1_{0}$ ordered FePd ferromagnets. Scr Metall Mater, 1995, 33: 1793-1805 


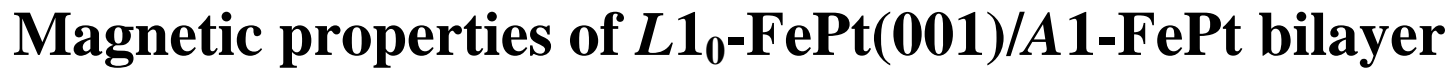 exchange springs
}

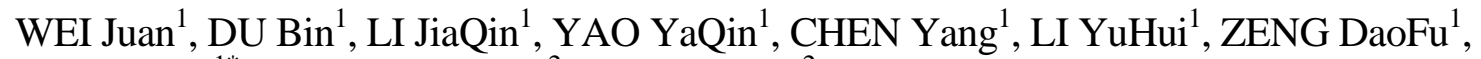 \\ LI GuoQing $^{1 *}$, ZHANG KunHua ${ }^{2}$ \& WEN Ming ${ }^{2}$ \\ ${ }^{1}$ School of Physical Science and Technology, Southwest University, Chongqing 400715, China; \\ ${ }^{2}$ Kunming Institute of Precious Metals, Kunming 650106, China \\ * Corresponding author, E-mail: gqli@ swu.edu.cn
}

Soft $A 1-\mathrm{FePt}(30 \mathrm{~nm})$ films were magnetron-sputtered on $\mathrm{MgO}(001)$ single-crystal substrates at an elevated temperature of $400^{\circ} \mathrm{C}$, and subsequently annealed at diffrent temperatures in range of $T_{\mathrm{a}}=\left[400^{\circ} \mathrm{C}, 700^{\circ} \mathrm{C}\right]$ for $6 \mathrm{~h}$ in order to turn them into hard magnets with different degrees of $A 1 \rightarrow L 1_{0}$ transformation. Then second layers of $A 1$-FePt with different thicknesses were covered at $100^{\circ} \mathrm{C}$ to obtain $L 1_{0}-\mathrm{FePt} / A 1-\mathrm{FePt}$ bilayer magnetic exchange springs with (001) textures. At $T_{\mathrm{a}} \leqslant 600^{\circ} \mathrm{C}$, the coverage of hard layer was $\sim 100 \%$, though the grain size increased with $T_{\mathrm{a}}$. The surface roughness reduced from 2.45 to $1.02 \mathrm{~nm}$ if the film was annealed at $T_{\mathrm{a}}=500^{\circ} \mathrm{C}$. The surface of hard layer flattened by annealing should benefit to improve the strength of interlayer exchange interaction. At $T_{\mathrm{a}}=500^{\circ} \mathrm{C}$ and $600^{\circ} \mathrm{C}, A 1-\mathrm{FePt}$ and $L 1_{0}-\mathrm{FePt}$ coexisted in the hard layer with an ordering degree of 0.61 and 0.84 , respectively. The direction of magnetic easy axis began to switch from in plane to out of plane at $T_{\mathrm{a}}=500^{\circ} \mathrm{C}$, indicating a vertical magnetocrystalline anisotropy was generated. The out-of-plane magnetization curves showed rectangle-like shapes at $500^{\circ} \mathrm{C} \leqslant T_{\mathrm{a}} \leqslant 600^{\circ} \mathrm{C}$, in spite of the soft phase left in hard layer, and a moderate coercive force of $\sim 5 \mathrm{kOe}$ was obtained at $T_{\mathrm{a}}=500^{\circ} \mathrm{C}$. This indicates the exchange interaction between coexistent two phases in hard layer was strong enough to pin the soft magnetic moments with the hard ones. At $T_{\mathrm{a}}=700^{\circ} \mathrm{C}$, the coercive force exceeded $20 \mathrm{kOe}$, which is too huge to be affected by exchange springs. By using the film of $T_{\mathrm{a}}=500^{\circ} \mathrm{C}$ as hard layer, $L 1_{0} / A 1-\mathrm{FePt}$ bilayer film with $20 \mathrm{~nm}$ thick soft layer kept rigid behaviors with almost the same coercive force of single hard layer, and respective reversal steps for soft layer and hard layer appeared in the magnetization curve if the thickness of soft layer was $30 \mathrm{~nm}$, with a reduced coercive force of $\sim 4$ kOe. As double shoulders should appear in the hysteresis loop if the thickness of soft layer exceeds twice of domain wall thickness of hard layer, i.e., the so-called interlayer exchange length, this indicates that the hard/soft interlayer exchange length was in the range of $20 \mathrm{~nm}<l_{\mathrm{ex}}<30 \mathrm{~nm}$, at least twice of ideal $L 1_{0} / A 1-\mathrm{FePt}$ exchange spring. The reason is due to that the coexistence of $A 1-\mathrm{FePt}$ and $L 1_{0}-\mathrm{FePt}$ in hard layers lowered the efficient uniaxial magnetocrystalline anisotropy. This lengthened distance of interlayer exchange could be maintained at $T_{\mathrm{a}}=600^{\circ} \mathrm{C}$. This is benefit to design the properties of magnetic springs for different applications.

doi: 10.1360/N972016-00592 